\title{
'It Has a Lot of Potential!': Use of Blockchain Technology for Education Records
}

\author{
Justyna Kaletka, Department of Informatics, Linnaeus University, Sweden \\ Andreas Raoul Herkommer, Department of Informatics, Linnaeus University, Sweden \\ Niki Chatzipanagiotou, Department of Informatics, Linnaeus University, Sweden
}

\begin{abstract}
The paper explores the perceptions of university students and their desired features on the use of blockchain technology for the management of education records. A literature review forms the theoretical basis of the research allowing to explore how education records are managed nowadays and the potential for the use of blockchain technology in this area. The theoretical framework is then used to discuss the research findings.

The research adopts the interpretive qualitative approach and collects data through a focus group interview with university students. Computer assisted thematic data analysis yields five key themes: current usage of university education records, understanding how blockchain works, sustainability of blockchain, security of blockchain and implementation of blockchain for education records. Participants were generally positive towards the use of blockchain for the management of education records and saw it as one of the potential future solutions. Nevertheless, they voiced some reservations regarding the high energy consumption, costs and security towards a possible use of the blockchain technology. Therefore, careful implementation would be needed, with increased focus on usability, solving some security and sustainability issues and ensuring a fair and transparent access model.

This research contributes to the current body of knowledge within informatics by empowering students to share their perception of possible development of student record systems based on blockchain technology. It also provides insights which can be used in the future to achieve a more user focused design of education records management systems.
\end{abstract}

\section{Introduction}

Nowadays Information systems (IS) and Information and Communication Technology (ICT) are fundamental for both organizations and individuals (Beynon-Davies, 2013). Subsequently, IS and ICT also became increasingly important for organizations in the education sector such as schools and universities. Many universities worldwide use modern IS and ICT to support their daily operations with the help of Student Information Systems (SIS) (Mukerjee, 2012), Student Information Management Systems (SIMS) (Jin et al., 2012), or Student Record Systems (SRS) (Strimbei et al., 2016).

One of the most important applications of ICTs at universities is the management of education records (Strimbei et al., 2016). The way universities currently manage the educational records of students with the current types of systems received increased critique in recent times. According to Jirgensons and Kapenieks (2018), the educational records often do not include information about the skills achieved by a student, mastery level and extracurricular activities and, therefore do not represent all relevant information required in the modern working world. Turkanovic et al. (2017) pointed out that many of those systems used by universities are set up in a way that education records are stored in databases which are only accessible by the students and staff of one university and therefore offer a lack of interoperability. In times, where students increasingly move between countries to achieve multiple different degrees, this often causes additional complications. Since education records need to be exchangeable between different institutions, paper certificates need additional effort for validation as they can be forged or manipulated (Grech and Camilleri, 2017).

The blockchain technology received an increased amount of interest (Chen et al., 2018) aiming to improve the challenges of increased academic mobility of students and lecturers, changed learning goals, as well as optimizing the administrative tasks related to the management of these education records. Blockchain is a decentralized data infrastructure, that can be used to store any type of assets or value such as property, shares, money and identity, while securing a maximum of security, trust and reliability (Open Data Institute, 2016; Chen et al. 2018). According to the Open Data Institute (2016), there are benefits both for organizations and people that could come from blockchain innovations. The recommendation is to use it in specific cases, where the blockchain technology can be used to overcome current problems and limitations. 
Regarding the possible use cases for applying the blockchain technology in the education sector and specifically universities, several initiatives have been carried through proof of concept and prototypes (Rooksby and Dimitrov, 2017; Turkanović et al., 2018; Kuvshinov et al., 2018). These education records include the performance of students during their education in the form of the attended courses/programs, achieved ECTS (European Credit Transfer and Accumulation System) points as well as the received grade and certificates. Main stakeholder groups of these systems include university administration staff, professors, IT maintenance staff and of course students. However, until now students' voice has not been prominent in the design discussions (Rooksby and Dimitrov, 2017; Turkanović et al., 2018; Kuvshinov et al., 2018). As this application of the blockchain technology is still in its infancy, we believe that active participation of stakeholders such as students can make an impact in how it will develop in the future.

This paper, thus, aims to explore university students' perceptions of the blockchain technology and its possible application for education records and, in consequence, explores possible desired features that exist from a student perspective to contribute to a more user focused development of blockchain solutions.

The paper is structured as follows. Following this introduction, the paper continues with section two, a brief literature review and the theoretical framework used in the research. Section three describes the paradigm, methodology and the methods of data collection and data analysis process. The findings are presented in section four along with a discussion. The paper concludes with section five which presents the conclusions of this research.

\section{Literature Review and Theoretical Framework}

The literature review formed the theoretical basis of this work by presenting key concepts which are currently involved in the management of education records in universities such as information systems (IS), information and communication technology (ICT) as well as SIS (Student Information Systems), SIMS (Student Information Management Systems), SRS (Student Records Systems) and digital certificates.

\section{Definitions of main concepts}

Education records have been defined in several ways by different government authorities and vary geographically. It is important to understand the scope and limitations of all these definitions, as the existing blockchain solutions for education records were developed around the world. A definition of education records, given within the Family Educational Rights and Privacy Act and adopted in this research, defines student education records as records, files, documents and other materials which contain information directly related to a student (US Department of Education, 2017). Since this research is conducted in Sweden, the researchers decided to mainly focus on education records which apply the European Credit Transfer and Accumulation System (ECTS) framework that is used in Sweden and creates a common standard among Higher Education Institution (HEI), such as universities, the European Commission and EU member states regarding the information that these education records store about the performance of a student.

\section{IS and ICT}

To understand the possible impact that the blockchain technology can have on university education records management, it is important to understand how ICT systems usually operate and what role they play in today's universities. According to Beynon-Davies (2013, p. 131), an information system is "a socio technical system, a communication system in which humans use ICT in support of coordinated and collaborative decision making and action". According to Dobre (2015), the wide implementation of ICT systems within universities started with the ICT boom at the end of the 90s. Subsequently, those systems could be regarded as the core of universities since they not only offer the management of education records, but also a wide number of additional functionalities which are essential to the management of universities (Mukerjee, 2012). Regarding the technical setup of those systems, Tang and Zhang (2009), Liu et al. (2010) and Jin et al. (2012) proposed a typically three-tier or n-tier architecture using several layers such as the interface, business and database layer operating on a client-server communication. According to Turkanovic et al. (2018) this often causes the following problems: First, the databases of those system are often only accessible by the administration staff of the university hosting those systems and often don't offer any interoperability with other universities and organizations. Second, students can only have access to the data but not own the data and therefor rely on the student record system to access their education records. Third, the data format in which the education records are stored often differ in standards in example languages and data format. Therefore, possibilities of how this traditional architecture of system store education records should be investigated.

\section{Blockchain Technology}

The blockchain technology gained worldwide attention in 2008 in connection with the introduction of the cryptocurrency Bitcoin (Grech and Camilleri, 2017). The features of the blockchain will be explained further in 
order to offer a foundation from a technical and a processual perspective on how the blockchain technology evolved. Additionally, the presentation of the blockchain features will allow a better understanding of the opportunities and limitations that blockchain technology offers for the management of education records. A blockchain is built of blocks that are chronological linked to each other and, therefore, form a chain of blocks as illustrated in the following Figure 1.

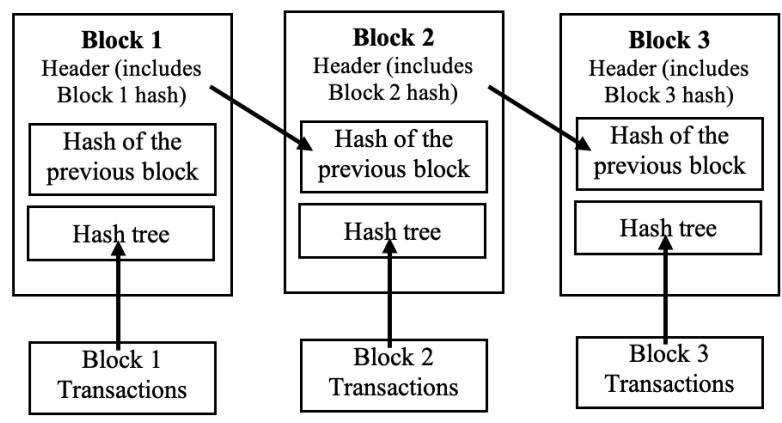

Figure 1. An example of blockchain structure (adopted from Nakamoto, 2008 cited in Mougayar and Buterin, 2016)

A block itself consists of a block header and a block body. The block header includes a block version, a Merkle tree root hash which represents the hash code of all transaction within the block and a timestamp (Zheng et al., 2017). In addition, the block header contains nBits which include the target threshold of a valid block hash, a random number called nonce which increases for every hash calculation and the parent block hash to link the block with its previous block (Zheng et al. 2017; Tinu, 2018). Transactions to be saved must reach a consensus within the blockchain, which is reached by the usage of a consensus mechanism. If the consensus is reached, the transaction is hashed into a block by a member of the network and the block added to the blockchain if most of the members of the network approves the block (Christidis and Devetsikiotis, 2016).

In order to be saved transactions must reach a consensus within the blockchain. This consensus is reached by the usage of a consensus mechanism. If the consensus is reached, the transaction is hashed into a block by a member of the network and the block added to the blockchain if most of the members of the network approves the block (Christidis and Devetsikiotis, 2016).

Compared to centralized systems such as a current student records system in which clients use the services provided by servers, SRS based on blockchain technology would rely on a decentralized peer-to-peer network in which the nodes represent a collective of local computers which are connected remotely and complete tasks or functions together as local ledgers (Mougayar and Buterin, 2016).

According to Zheng et al. (2017), blockchains are currently categorized in three types: public blockchains, private and consortium. Public blockchains basically represent the original blockchain characteristics as they are described by Nakamoto (2008 cited in Mougayar and Buterin, 2016). Public blockchains can be joined and left at will without the need to meet a defined set of predetermined criteria while also staying anonymously, only represented by the public and private key. In addition, all members of the blockchain can participate in the consensus process such as Proof of Work (PoW) in which the nodes performing the consensus mechanism get rewarded for their efforts. In this way the records of the blockchain are stored in every node and therefore are almost impossible to be manipulated (Mougayar and Buterin, 2016; Zheng et al., 2017). Furthermore, the records of each block are visible to all members of the blockchain which allows a very high level of transparency.

In comparison to public blockchains, private and consortium blockchains are fully or partially centralized (Zheng et al. 2017) and have increased in popularity. In this case, the permission for reading the transaction are granted by one single organization or between the consortium participants (Dib et al. 2018). Therefore, there is less anonymity compared to the approach of public blockchains since the identity of the network members need to be known in advance in order to grant them permission (Zheng et al. 2017). Consequently, this can increase the trust into the blockchain since the members of the blockchain are known and the private data is more secure. Another difference is that only one central node or a set of predefined nodes run by the organization has the right to perform the consensus process (Zheng et al. 2017). In this way, private blockchains achieve a much higher efficiency since the used algorithms require less computing power a higher transaction throughput can be achieved (Dib et. al. 2018, Zheng et al. 2017). Hence, private and consortium blockchains are more suitable for organizations that want to retain the control about the participants of their blockchain and need to perform transactions in a more efficient way.

Grech and Camilleri (2017) argued that the blockchain technology could enable the self-sovereignty of students regarding their academic records. Turkanovic et al. (2018, p. 5112) who also introduced the blockchain EduCTX, 
which enables "a globally unified viewpoint for students and higher education institutions (HEIs), as well as for other potential stakeholders", argue that the time when education records that were only stored in the infrastructure of institutions could come to an end through blockchain. This also offers the possibility for certain stakeholders to directly and automatically verify the validity of certificate and academic records against the blockchain. Therefore, there will be no need to contact the institutions which originally issued them (Sharples and Domingue, 2016; Grech and Camilleri, 2017).

Looking at the multitude of current prototype solutions, there is still no clear consent about the question if blockchain needs to be open, private or consortium based for educational records. On the other hand, MIT Media Lab team (2016) has pointed out that the blockchain cannot be considered a simple solution able to fix everything with today's credentials. Different design decisions can have effects that reach far beyond the issuing of a certificate or storing an education record. MIT Media Lab recommends thoughtful discussion about these implications.

\section{Methodology and Methods}

\section{Paradigm and Methodological Approach}

The research adopts the interpretive paradigm which reflects our interest in exploring people's perceptions and understandings. Interpretivism implies that reality is socially constructed and can be approached and understood through the meanings people give to phenomena (Klein and Myers, 1999; Walsham, 2006). The interpretive paradigm is, then, considered suitable since the context of this research is the university students and what meanings they assigned to an information system which uses blockchain technology to manage university education records. The qualitative methodological approach is chosen because, according to Patton (2015), it allows researchers to gain an inductive and holistic understanding of human experiences and, therefore, supports the understanding of university students' perceptions and desired features regarding the application of blockchain technology for the management of their education records.

\section{Methods of Data Collection}

The empirical material was collected through a pilot interview and a focus group discussion among university students enrolled at Linnaeus University, which is in the Swedish municipality of Växjö. The discussion was facilitated using an interview script accompanied by a presentation about blockchain.

First, a pilot interview, which lasted for an hour, was carried out with an interviewee at Linnaeus University Library in Växjö campus matching the target group of the research. According to Majid et al. (2017), a pilot interview can be used to test out if the presentation and the questions that were prepared for the focus group is adequate or further adjustments are necessary. The pilot interview was recorded with the mobile phones of the researchers and later was transcribed. The transcribed feedback gathered from the pilot interview was later reviewed by the researchers and was used to make improvements and adjustments to the presentation and the interview script that was meant for the focus group.

Second, a small group discussion was facilitated by the focus group technique in order to collect empirical data to fulfil the aim of the research. A focus group is mainly based on insights that emerge from a facilitated group interview of a group with similar characteristics and power relations; in this case a group of university students (Cook and Crang, 2007). The focus group consisted of six participants that were selected purposefully (Creswell, 2014). Therefore, the participants were all currently studying at the Linnaeus University and had experience of using student record systems, as well as basic knowledge and understanding of ICT and IS. The focus groups allowed us to explore what students thought about the phenomenon under study by setting up a situation in which a group of people met to discuss about their experiences and thoughts (Cook and Crang, 2007). The focus group interview took place in a study room at the library of Linnaeus University in Växjö. The room offered place for eight people and included a TV screen which was used to hold a short presentation at the beginning of the focus group interview, a whiteboard which was used to place the different post-it notes that covered the thoughts of the participants and a table and chairs so that the participants could sit comfortably and interact with each other. The focus group interview lasted about three hours. The aim was to generate a good discussion on the topic of the application of blockchain technology in relation to the management of university education records among the participants. However, we, the researchers, had at the same time to manage the course of the discussion and to encourage the participants to engage and stay focused on the study purpose. The focus group was audio recorded with the consent of the participants by using the mobile phones of the researchers. The recordings were then transcribed.

\section{Method of Data Analysis}

For the analysis of the empirical material we followed thematic analysis, which is the process of identifying themes in the collected data and unfolds in six steps (Creswell, 2014): First, the raw data which was collected during the 
focus group interview was organized and prepared to be transcribed. Automated transcription software Trint has been used to create a first text version of the interview data from the voice recording. The automated transcription was then corrected by one of the researchers to ensure exactness. Second, the transcribed data was read several times to get a general sense of the collected empirical material. Third, the transcribed data was coded. That means, the transcribed text was separated into chunks that included similarities and was classified into some initial categories. Fourth, the categories were scanned for overlapping or irrelevant material. The categories were, then, lifted to the level of identified themes by bringing similar categories together. Fifth, the identified themes were interrelated. Finally, interpretations of the findings were made. To aid the analysis of the collected data, MAXQDA Analytics Pro 2020 has been used. Transcribed interview data has been loaded into MAXQDA and then coded and analysed.

For the research, we followed ethical guidelines applied in social sciences (Walsham, 2006). Participants were voluntarily involved and were able to withdraw at any stage of the research. Participants were informed of their rights, their role of participation and the focus group interview was conducted after getting written informed consent forms from them. Confidentiality was assured and personal information of the participants was not revealed.

\section{Findings and Discussion}

The data analysis process explained in previous section yielded the five themes: current usage of education records, understanding how blockchain works, sustainability of blockchain, security of blockchain and implementation of blockchain for education records.

\section{Theme 1: Current usage of education records}

Participants shared their different experiences with regards to the use of education records in the form of certificates and diplomas. The research participants were coming from Sweden, USA, China, Romania, Malaysia and Serbia. The researchers conducting the focus group interview come from Germany and Poland. Student 5 said about the education records management in Sweden: "Like I said in Sweden [the system] it's kind of centralized. So, like my grades are up there. I have a paper copy but it's like up there so I can just, like, download it whenever I want. If I want to apply abroad or something and they wouldn't be, you know, in our system, then I can just download it and send it instead of, I don't know, going to my university and paying money."

Most common situations, where students needed to use their educational records, were applying for university, applying for a job and extending a visa. Student 1 shared his/her experience of using student education records systems at the university: "When the bachelor's graduation is finished and all the things appear on the website, you have different functions there. They would have a course service survey like to. The teacher survey me and then, like, students' survey, so I'll finish on the website." Participants also discussed the use of certificates to apply for university including studying abroad.

One of the key aspects discussed by the student was granting access to their education records to other people than the students themselves, such as parents or sponsors. Another aspect was not having a need to handle any documents themselves due to fully digital solutions being in place. According to participant 3 some universities in USA have a sophisticated permission management set up for student, parents and sponsors to see the education progress: "That's something I think my father was talking to me about at the one of the more premium 'unis' back home, that the parents or sponsor can actually follow the grades of the student. They see everything and access it anytime and the school actually allows that. Also, if you miss out of class everything is there. So basically, if you mess up your parents or your sponsor will know, so that's pretty cool in a technological standpoint but as a student you bet."

Several participants noted a critical shortcoming of paper-based certificates and credentials - the possibility of forgery. Student 2 shared: ""Hey, I want to see your results" because that's the other thing because it is also possible as a student. Let's say my sponsor is from my country right. So, I'll say okay, I'll print it out in an hour, change them in Photoshop and I'll send it to them. So that's another thing that can happen."

\section{Theme 2: Understanding how blockchain works}

As blockchain technology is a relatively new and complex concept, an important part of the focus group interview was spent on reviewing support presentation and videos and discussing the principles behind blockchain design.

Student 3 shared his/her view on the design of blockchain: "I wouldn't say it's super hard to understand. How it was explained was pretty all right. But, like, you say you need to imagine the whole key and the fact that you don't really see all, even though the information is public, you don't really have access to the data. I think if people understood that then maybe they could agree on, yeah, it's actually quite secure and then it's not really that complicated." 
Student 1 shared his/her view on blockchain's immutability as well as the validation of credentials in time: "The thing is another problem for this, if it's like it exists forever, so there will be a problem that we have a certain period of time with the validation. If you pass the course like 10 years ago, it might not make sense anymore. There are some exams that are only valid for two years. [...] If you take the test, like, say the programming course in the 1970's, I don't think it's valid until now. It'll make no sense because the domain of knowledge it's totally changed."

Students mostly saw blockchain mining as a way to make easy money through crypto currencies however one of the students felt that the use of blockchain education apps could resolve some of the issues described later on in the findings. Student 1 said: "I used to dig the coins but after that they have some Chinese company like they can let you dig on the phone. But actually, right now I think it's not like you are digging, it's like they are using your power, so more like it's a good way, like to solve the energy problem that all of the students support power and energy."

\section{Theme 3: Sustainability of blockchain}

Focus group interview participants were interested in several sustainability related aspects of possible applications of blockchain for education records, specifically about the possible environmental impact.

Based on the discussion about the consensus mechanisms, the participants understood that the blockchain technology requires high computing powers which can affect the costs of a blockchain system and could have environmental effects. Student 2 argued that: "I agree with [student 1], so provided that it's implemented, it has potential. Is how it's going to implement it or how the blockchain is being used. And also, we have to think about resources in terms of energy and people to validate and what not. That's about it and, if I could still just use, run a script and then copy, paste and what's the point of validating anyway? So, I think a lot of things needs to be thought through I guess, then. But it will be implemented because this is the future as they say. " Especially student 3 saw the consensus mechanism quite sceptical by saying "for me obviously the environmental factor would be a big thing [...]".

Student 4 saw blockchain solutions in a sceptical light, sharing that in his/her opinion the solutions are: "They're not scalable right now." Student 2 noticed a scalability aspect linked to both energy consumption and security: "Because over time I would also assume that the longer the hash, the bigger the blocks become, the more power it would need. So that also means that with over time we need way more power. Then, it is no longer a sustainable even though it is very, very secure."

Student 3 noticed the cost as a factor that might influence the universities in deciding the future solutions: "Because I think if we're going for hardware, I don't think universities would spend that kind of money." Student 5 shared his/her view of the costs of switching to another solution for students: "A smooth transition from one to another, so that it doesn't take too much time to get used to the new system, cause that's going to be annoying, just another thing to learn."

\section{Theme 4: Security of blockchain}

Student 3 said that: "You need to imagine the whole key and the fact that you don't really even though the information is public you don't really have access of data." Student 4 was of opinion that it's important that the whole system of education records management is fair. He/she emphasized the impact of grades and certificates for participation in job recruitment and possible effect on student's career as a whole: 'If someone that doesn't know anything changes their own grades so that they match my grades which probably are higher. I'm still like in a bad position because they are in the same position as me to a new recruiter for example. It's something to have in the back of your head about the security of the grades."

Students had mixed opinions on who should be the blockchain validation authority. Student 4 said: "I think it should not be a public information to know the institute that's going to validate that certificate. Because I think that's a bit risky. So, it should be maybe selected. Select a different place every time, but then again that's the problem with a computing power. It shouldn't be like easy to know who's doing what."

Students were discussing at length that the education management systems as managed by universities or government agencies are highly political. The same could happen with systems based on blockchain. Participant 3 said: "So, the person with the super computer rules the world. Is still not it's still on balance in a way."

\section{Theme 5: Implementation of blockchain for education records}

After the discussion regarding the different consensus mechanisms, the students were shown a video and a slide regarding different types of wallets which are used for different blockchain applications. The overall perception of the students was that the basic web wallets would be a preferable option, as they are very similar to the current web interfaces of SRS. Using a hardware wallet seemed like an unnecessary complication. The reason for that can 
be seen in the positive perception the students had about the current system applied in Sweden, Ladok. According to student 1: "I never had any issue until now with Ladok. It is also easy to use, always available and it's operating since a long time". Nevertheless, the option of an app wallet also received mainly positive feedback. Student 3 called it a "more youth-of-today-friendly", but had some concerns regarding the security which feels more "iffier". Additionally, both student 5 and student 3 would like to be able to download a backup from the app which they could save. Student 5 stated: "I would like to have a way of creating back-ups from the data on the app and download my records and certificates." The USB wallet did not convince the students. As an example, student 6 stated that he/she would regard a hardware wallet as not very practical and, he/she would fear to lose the device stating: "To be honest, I lost more USBs than I would like to admit". There were also doubts of the students that a rollout and maintenance of hardware wallets would be achievable for the universities. Student 4 mentioned that he/she would expect high costs for the universities: "I think it would be difficult to implement but also you would have to give every student a USB device and track them."

Asked about the way a blockchain solution for educational records should be set up, the majority of the students agreed in favour of a permissioned blockchain, which only allows verified members to be part of a blockchain. "I think if the blockchain were to be public, you know it would need heavy encryption and anonymization to protect individuals' information [...] So I think only certain institutes and the students should have access." Student 5 also preferred a permissioned blockchain: "Well for me it's most important that only certified people and institutions would be in the network, so I think the permissioned version would be the way to go."

Since the majority of the participants were in favour of a permissioned blockchain, it comes as no surprise that the majority also preferred for the blockchain to be hosted and blocks to be validated by certain institutions and their members. Student 5: "Yes, since this combines the advantages of both the other types giving more computing power than a private blockchain and more security than a public one. [...] I suppose this is the closest thing to a centralized system and I imagine would make it easier to go between universities for studying. I wonder if such places as CSN for example would also get some sort of restricted access. To validate that you are studying."

Regarding the preferred consensus mechanism, the students were most in favour of proof of authority. Student 1 said: "This sounds like the most applicable one so far, maybe because it is similar to what we have now."

Participants were generally positive towards the future uses of blockchain for education records. Student 4 shared his/her perception of blockchain's future potential: “I think it has a lot of potential and it's definitely going to be implemented in the future, but it's just a matter of how efficiently you manage to implement it and how securely you can do it. But it has way more potential than the current systems, so I think, if you are careful with the details, it can be a blast and it can be something really good."

In the perception of the participants, the implementation of blockchain for education records should be performed in a careful and well-planned manner. In students' opinion blockchain implementation depends on the maturity of available hardware: Student 2 said: "Until the technology is more efficient than possible." Student 2 also said that: So I think a lot of things needs to be thought through I guess, then. But it will be implemented because this is the future as they say."

\section{Discussion}

The perception of the participants of this research was that blockchain is generally secure. This was clearly an advantage of this technology over current solutions which were seen as susceptible to fraud, mistakes or even hacking. The manipulations mentioned included the hacking of a SRS database but also the tampering of certificates which were easy to manipulate as not handed in a digital form. A disadvantage of current SRS which also have been introduced in the literature by Grech and Camilleri (2017) and Chen et al. (2018). Students also saw the potential in the blockchain technology to allow bigger networks of universities which could reduce the lack of interoperability which was also mentioned by several researchers (Grech and Camilleri, 2017; Turkanovic et al., 2017; Chen et al.,2018). In this regard, the students shared multiple experiences where they had to take over the exchange of documents and how annoyed they were by the amount of time it took them. However, as opposed to traditional database systems, blockchain has a significantly more complex design. Therefore, it is harder to be understood and trusted by the potential users.

Throughout the focus group, it was obvious that it took some time for the students to understand the different blockchain attributes. Considering recommendation of Gurguc and Knottenbelt (2018) who suggested investing in blockchain education through design-thinking and offering easily accessible and comprehensible educational materials would help in further understanding of how blockchain works and therefore reduce possible reservations by the potential users. When presented with support education materials such as a presentation and videos, participants were able to specify which blockchain design features are the most important for them. In their perception, an education records management solution based on a permissioned blockchain seemed more trustworthy and secure than a permissionless blockchain. This means that only authenticated users should have access to the system and that the validation is only performed by members whose identity is known to the system. 
This perception matches the findings of Rooksby and Dimitrov (2017) regarding how important trust is in the evaluation of grades and for the status of a university. Based on the feedback of the participants, a validation by an authority seemed more secure and trustworthy than a validation by anonymous validating members. Hence, in this question the students were quite opposed to the original blockchain definition by Nakamoto, where the members of the network are anonymous and the validation can be performed by anyone based on the computing power (Nakamoto, 2008 cited in Mougayar and Buterin, 2016). Furthermore, the students favored private blockchain solution for each university or a consortium blockchain of universities and other organizations since it seemed more secure regarding their data and also because it is a more trustworthy setup as an open blockchain were the members are anonymous. This further shows how important trust is for students when it comes to sensitive data such as education records since this approach would basically limit the interoperability between universities.

The participants of the study shared a concern where that potential solutions based on blockchain could have issues with scalability, as many of the initial design decision would influence how the system works across different institutions and geographic locations. Another topic which was seen cautiously by the participants was the immutability of blockchains. This is one of the unique characteristics distinguishing blockchain from other technologies, which means that all data is stored indefinitely and all transactions leave a trace since they are documented within a block of the blockchain (Mougayar and Buterin, 2016). If the validation of the record would have been false, such as in a case of a failed course or exam, a new block would need to be added. Students seemed to percept this feature as difficult since eventually incorrect records would be added to the blockchain. To ease that concern additional measures would need to be taken to ensure that only the valid record is displayed in the transcripts. Especially at the beginning until the solution matures. Furthermore, staff would need to be trained to limit possible mistakes since each validation could cost valuable energy an aspect which was also seen critical by the students. Therefore, implementing an application for education records would need to ensure that updated records are not displayed in real time as soon as a block with updated information is available to allow for correction of human errors.

Sustainability aspect of blockchain solutions for management of student education records was seen by the participants as vital. Specifically, in the context of the three types of blockchain solutions (public/consortium/private), public blockchain solutions were perceived as not friendly to the environment by the students. As described in the literature review, those types of blockchain mainly use a PoW (Proof of Work), which is very energy consuming, since the validation is based on computation power that is also used for mining the related cryptocurrency (Zheng et al. 2017). Due to a multitude of transactions in a public blockchain system, a solution becomes less and less sustainable over time due to the increasing length of a single block. This attribute of high energy consumption raised strong concerns by the participants regarding the PoW. A perception which could further explain the more favourable opinion of the students that a Proof of Authority (PoA) or Distributed Proof of Stake (DPoS) would be more suitable. From the solutions that have been presented in the literature review, only EduCTX and its DPoS mechanism match this desired feature of the students. In addition, distributing the computing load over multiple devices through mobile apps could solve some problems related to energy consumption as well as help validate transactions fairly. Participants added that it might take more time until devices which consume less energy become affordable or available for use. Until then, a PoA or DPoS where the validating nodes of the network could be spread among different countries as proposed by Turkanovic (2017) seem like a viable option. Consistently with statement from MIT Media Lab team (2016) that any potential blockchain solution has to be thoughtfully discussed, the participants of the research were ready to delve into details of possible information systems' designs and considerations.

Blockchain was perceived by participants as having a high potential for use for education records management when ensuring a careful implementation. Students have described blockchain as "the future", however many design decisions would have to be made to fulfil its potential. Participants noted some risks to the implementation such as investment costs for universities, specifically when deciding on purchasing new hardware. Blockchain hardware used for PoW based mining, such as Bitcoin mining is usually expensive and needs replacing frequently (Lund et al., 2019). This concern not only came up regarding the hardware to validate the transactions but also when the students where asked about the blockchain wallets. The students expected that this could create costs for the universities which would have to buy and maintain them. Finally, students noticed potential switching costs for themselves, when they would need to get used to a new solution. An example can be seen in the way they rejected the possible effort of using hardware wallets to access their education records as they considered them as too laborious. According to the literature, Barber et al. (2012) and Krombholz et al. (2017) described the advantages of such hardware wallets but in the students' perception that would be too much effort. This further indicates that data security is important to them but even more so is the usability they expect. Making sure that user desired features and preferences are incorporated into the IT development process is one of the strategies of increasing system adoption and user satisfaction (Liu et al., 2010). Next section will describe the students' desired features in more details. 
Impact of including students' feedback and perceptions to analyse the desired features of a potential blockchainbased solution could influence the adoption of this technology in other areas or educational contexts. Understanding scenarios specific to university students, their potential needs, requirements and education system usage patterns could help form the basis for further development in this area. The initial reflection of the research participants was that education records are needed in many everyday life contexts. Availability of good quality student information systems can have a profound impact on students' educational life and future career. Providing a fair and error-free solution would mean that students can access their information no matter where they live and work. This also means that a new solution based on blockchain ideally should be easy to use and provide a webbased interface that could be operated on different devices and did not require special hardware. Furthermore, participants wanted to know who has access to their education records and why. Ideally, they should be able to decide to share their data with others on demand or share their educational journey. In most countries that participants had experience with, students are not involved in handling of education records and they are just consumers of the information or some services provided by the university. As traditional education records systems are vulnerable and susceptible to forgery or hacking attacks, blockchain provides a promising vision of cryptographically secured data. This feature might come at a cost of higher computing desired features. Students expect a blockchain based education records management solution to be sustainable and have seen the sustainable energy consumption as one of the key decisive factors. According to the research participants' potential solutions based on blockchain could have issues with scalability, which is consistent with the findings of Walport (2016) and Rana et al. (2019).

To summarize, the participants of this research perceived potential blockchain-based education records management systems in generally positive light. Students saw high capability of blockchain, especially with careful implementation, focused on ensuring sustainability and fairness. A blockchain solution that enables user authentication (permissioned), established within a university (private) or between several universities and other organisations (consortium) with Proof of Authority (PoA) as consensus mechanism could fulfil their desired features. Certainly, a more distributed and transparent solution, with clear data sharing rules and free of political influence could benefit many user groups, not only students.

\section{Conclusion}

This paper has presented the perceptions of university students towards the potential use of blockchain technology for the management of education records and identified university students' desired features that a potential application of blockchain technology for this purpose should have. For achieving the aim of the research, the field of education records and related systems which nowadays are used in universities, the blockchain technology and its current application in the area of concern have been explored. The data collection was conducted among six university students that are enrolled at Linnaeus University in Sweden. The empirical material was collected through a focus group interview allowing a better understanding of the stakeholders' perceptions and desired features. The gathered data was thereafter analysed and discussed in order to generate the research findings to give students a voice when it comes to the development of new SRS that could potentially use the blockchain technology.

The findings showed that the general perception of students towards the application of blockchain technology was quite positive, since it could solve certain issues, which are still present according to the literature and students views. These include lack of interoperability, control over students' own records and security. Nevertheless, the students showed that especially security is not the only factor influencing their perception. When it comes to their grades the students expect that the validation is carried out by authorities and not anonymous miners. Furthermore, they were critical towards the high energy consumption of the certain blockchain types and consensus mechanisms. In a time were sustainability became increasingly important regarding the global climate crisis, the high energy consumption that some consensus mechanisms require for the validation of new blockchain blocks was met with serious doubt by the students. Moreover, it became quite obvious that the usability is a key factor for students. To achieve the user-acceptance of SRS which uses the blockchain technology, the application would need to match these perceptions of the students. Furthermore, an SRS which uses the blockchain technology, would need to compete with a user-friendly experience of the current SRS such as Ladok. The findings also showed that user experience focused, a user-friendly interface and functionality represent key desired features for students regarding the application of blockchain technology. Subsequently, the students opted for web-based wallet, which means that they would prefer the current way of accessing their grades via a web-browser. Additionally, the students preferred that the hosting and the validation of the educational record would be carried out by authorities such as universities. They also required more control over the educational records to share access to their information with other people or organizations such as family, sponsors or companies.

This research contributes to the current body of knowledge within informatics by empowering important stakeholders, such as students, to share their perceptions and desired features regarding the potential use of blockchain technology in the management of education records. It enriches and extends the existing body of 
knowledge and provides some valuable insights for the use of an innovative technology, such as blockchain in the management of education records. The research also contributes to a more user focused design of blockchain applications since important stakeholders and their perceptions and desired features have not been really included so far in this field. In this way, a higher user-acceptance and interest into the blockchain technology and its application can be achieved.

Since this research only included students' perceptions, further future research can be conducted to include additional stakeholder groups of this area such as administration staff, professors and IT maintenance staff. In this way a richer picture of desired features could be achieved leading to a deeper understanding of the desired features of Student Record Systems (SRS) based on blockchain technology. Finally, the topic of legal component of blockchain use for education records management can be examined in the future to determine how country specific requirements could be fulfilled in a unified solution with user experience, security and sustainability in mind.

Acknowledgments. We would like to thank Professor Anita Mirijamdotter and Associate Professor Päivi Jokela for their constructive feedback. Many thanks to the research participants, the university students, who willingly participated in the process of data collection. Thank you reviewers for your helpful comments.

\section{References}

Barber, S., Boyen, X., Shi, E. and Uzun, E., 2012. Bitter to Better - How to Make Bitcoin a Better Currency. In: D. Hutchison et al., eds. Financial Cryptography and Data Security, Lecture Notes in Computer Science, vol. 7397. Berlin: Springer, pp. 399-414.

Beynon-Davies P., 2013. Business Information Systems. New York: Palgrave Macmillan.

Chen, G., Xu, B., Lu, M. and Chen, N.S., 2018. Exploring blockchain technology and its potential applications for education. Smart Learning Environments, [e-journal] 5(1), pp. 1311-1320. https://doi.org/10.1186/s40561-017-0050-X.

Christidis, K. and Devetsikiotis, M., 2016. Blockchains and Smart Contracts for the Internet of Things. IEEE Access, [e-journal] 4, pp. 2292-2303. https://doi.org/10.1109/ACCESS.2016.2566339

Cook, I. and Crang, M., 2007. Doing Ethnographies. London: Sage.

Creswell, J. W., 2014. Research Design: Qualitative, Quantitative and Mixed Methods Approaches. London: Sage.

Dib, O., Brousmiche, K., Durand A., Thea, E., Ben Hamida E., 2018. Consortium blockchains: Overview, applications and challenges. International Journal on Advances in Telecommunications, [e-journal] 11 (1\&2), pp. 51-64. Available at: <https://hal.archives-ouvertes.fr/hal-02271063/> [Accessed 13 February 2019].

Dobre, I., 2015. Learning Management Systems for Higher Education: An Overview of Available Options for Higher Education Organizations. Procedia - Social and Behavioral Sciences, [e-journal] 180, pp. 313-320. https://doi.org/10.1016/j.sbspro.2015.02.122.

Grech, A. and Camilleri, A. F., 2017. Blockchain in Education. JRC Science for policy report. [e-book] Luxembourg: Publications Office of the European Union. https://doi.org/10.2760/60649 .

Gurguc, Z. and Knottenbelt, W., 2018, Cryptocurrencies: Overcoming Barriers to Trust and Adoption. [pdf] London: eToro - Imperial College London Consultants. Available at: $<$ https:/www.imperial.ac.uk/media/imperial-college/research-centres-and-groups/ic3re/cryptocurrencies-overcoming-barriers-to-trust-and-adoption.pdf > [Accessed 16 January 2020].

Jin M., Qiu C., Li J., 2012. The designment of student information management system based on B/S architecture. In: IEEE (Institute of Electrical and Electronics Engineers), 2nd International Conference on Consumer Electronics, Communications and Networks (CECNet.). Yichang, China, 21-23 April 2012, pp. 2153-2155. https://doi.org/10.1109/CECNet.2012.6201529.

Jirgensons, M. and Kapenieks, J. 2018. Blockchain and the Future of Digital Learning Credential Assessment and Management. Journal of Teacher Education for Sustainability, [e-journal] 20(1), pp. 145-156. https://doi.org/10.2478/jtes-2018-0009.

Klein, H. K. and Myers, M. D., 1999. A Set of Principles for Conducting and Evaluating Interpretive Field Studies in Information Systems. MIS Quarterly, [e-journal] 23(1), pp. 67-93. https://doi.org/10.2307/249410 .

Krombholz, K., Judmayer, A., Gusenbauer, M. and Weippl, E., 2017. The Other Side of the Coin: User Experiences with Bitcoin Security and Privacy. In: J. Grossklags and B. Preneel, eds. Financial Cryptography and Data Security. Berlin: Springer, pp. 41-59. https://doi.org/10.1007/978-3-662-54970-4_33.

Kuvshinov, K., Nikiforov, I.V., Mostovoy, J., Mukhutdinov, D., Kirill, A. and Podtelkin, V., 2018. Disciplina: Blockchain for Education. [online] Available at: <https://disciplina.io/yellowpaper.pdf $\geq$ [Accessed 13 February 2019].

Liu Z.; Wang H.; Zan H., 2010. Design and Implementation of Student Information Management System. In: IEEE (Institute of Electrical and Electronics Engineers), International Symposium on Intelligence Information Processing and Trusted Computing (IPTC). Huanggang, China, 28-29 October, pp. 607-610. [online] https://doi.org/10.1109/IPTC.2010.160. 
Lund, E. Jaccheri, M., Li, J., Cico, O. and Bai, X., 2019. Blockchain and Sustainability: A Systematic Mapping Study. In: Association for Computing Machinery (ACM), Proceedings of the 41st International Conference on Software Engineering: Companion Proceedings. Montreal, Canada, 27 May 2019. https://doi.org/10.1109/WETSEB.2019.00009 .

Majid, M., Othman, M., Mohamad, S., Lim, S. and Yusof, A., 2017. Piloting for Interviews in Qualitative Research: Operationalization and Lessons Learnt. International Journal of Academic Research in Business and Social Sciences, [e-journal] 7(4). http://doi.org/10.6007/IJARBSS/v7-i4/2916 .

MIT Media Lab, 2016. What we learned from designing an academic certificates system on the blockchain. [online] Available at: <https://medium.com/mit-media-lab/what-we-learned-from-designing-an-academiccertificates-system-on-the-blockchain-34ba5874f196> [Accessed 28 February 2019].

Mougayar, W. and Buterin, V., 2016. The business blockchain: promise, practice, and application of the next Internet technology. New York: Wiley \& Sons.

Mukerjee, S., 2012. Student information systems - implementation challenges and the road ahead. Journal of Higher Education Policy and Management, [e-journal] 34(1), pp. 51-60. https://doi.org/10.1080/1360080X.2012.642332 .

Open Data Institute, 2016. Applying blockchain technology in global data infrastructure. [online] Available at: $<$ https://theodi.org/article/applying-blockchain-technology-in-global-data-infrastructure/> [Accessed 21 January 2019].

Patton, M. Q., 2015. Qualitative Research \& Evaluation Methods: Integrating Theory and Practice. 4th ed. Thousand Oaks, California: Sage.

Rana, R.L., Giungato, P., Tarabella, A. and Tricase, C., 2019. Blockchain Applications and Sustainability Issues. Amfiteatru Economic, [e-journal] 21(Special Issue 13), pp. 861-870. https://doi.org/10.24818/EA/2019/S13/861.

Rooksby J., Dimitrov K., 2017. Trustless Education? A Blockchain System for University Grades. In: DIS2017 (Designing Interactive Systems 2017), New Value Transactions: Understanding and Designing for Distributed Autonomous Organisations. Edinburgh, U.K.., 10 June 2017. [online] Available at: $<$ http://johnrooksby.org/papers/DAOworkshop_rooksby.pdf $>$ [Accessed 16 January 2020].

Sharples, M. and Domingue, J., 2016. The Blockchain and Kudos: A Distributed System for Educational Record, Reputation and Reward. In: K. Verbert, M. Sharples, T. Klobučar, eds. Adaptive and Adaptable Learning, ECTEL 2016. Lecture Notes in Computer Science, vol 9891. Springer, Cham. https://doi.org/10.1007/978-3-31945153-4 48

Strimbei, C., Dospinescu, O., Sinu, R, Nistor, A. 2016. The BPMN Approach of the University Information Systems. Ecoforum, 5, pp. 181-193.

Tang, Y., Zhang, Y., 2009. Design and implementation of college Student Information Management System based on Web Services. In: IEEE (Institute of Electrical and Electronics Engineers), International Symposium on IT in Medicine \& Education. Jinan, Shandong, China, 14-16 August 2009, pp. 1044-1048. https://doi.org/10.1109/ITIME.2009.5236230.

Tinu, N.S., 2018. A Survey on Blockchain Technology- Taxonomy, Consensus mechanisms and Applications. International Journal of Computer Sciences and Engineering, [e-journal] 6(5), pp. 691-696.

Turkanović, M., Hölbl, M., Košič, K., Heričko, M. and Kamišalić, A., 2018. EduCTX: A Blockchain-Based Higher Education Credit Platform. IEEE Access, [e-journal] 6, pp. 5112-5127. https://doi.org/10.1109/ACCESS.2018.2789929.

US Department of Education, 2017. Family Educational Rights And Privacy (FERPA). [online] Available at: $<$ https://studentprivacy.ed.gov/node/548/> [Accessed 20 January 2020].

Walport, M., 2016. Distributed ledger technology: Beyond block chain. A report by the UK Government Chief Scientific Adviser. UK Gov. Off. Sci. [online] Available at: <https://assets.publishing.service.gov.uk/ government/uploads/system/uploads/attachment_data/file/492972/gs-16-1-distributed-ledgertechnology.pdf $\geq$ [Accessed 13 February 2019].

Walsham, G., 2006. Doing interpretive research. European Journal of Information Systems, [e-journal] 15(3), pp. 320-330. https://doi-org.proxy.lnu.se/10.1057/palgrave.ejis.3000589.

Zheng, Z., Xie, S., Dai, H., Chen, X. and Wang, H., 2017. An Overview of Blockchain Technology: Architecture, Consensus, and Future Trends. In: IEEE (Institute of Electrical and Electronics Engineers), 2017 IEEE International Congress on Big Data (BigData Congress), Honolulu, USA, 25-30 June 2017, pp. 557-564. https://doi.org/10.1109/BigDataCongress.2017.85. 